\title{
Low-temperature antihydrogen-atom scattering
}

\author{
S Jonsell \\ Department of Physics, Umeå University, SE-90187 Umeå, Sweden
}

\begin{abstract}
A simple method to include the strong force in atom-antiatom scattering is presented. It is based on the strong-force scattering length between the nucleon and antinucleon. Using this method elastic and annihilation cross sections are calculated for hydrogen-antihydrogen and helium-antihydrogen scattering. The results are compared to first-order perturbation theory using a pseudo potential. The pseudopotential approach works fairly well for hydrogen-antihydrogen scattering, but fails for helium-antihydrogen scattering where strong-force effects are more prominent.
\end{abstract}

Key words: Antihydrogen, antimatter, scattering, annihilation.

PACS: 36.10.-k, 34.50.-s

Antimatter in contact with matter causes annihilation - a violent process where mass is converted to energy. This is well known, even among people who never have studied physics antimatter annihilation seems to stimulate the imagination. In popular films and literature antimatter is used as e.g. an energy source, fuel for space ships, or a weapon. However, since the energy required to create antimatter outweighs the energy created by annihilation by many orders of magnitude, neither of these applications are possible in reality. More realistically, antimatter may have applications in medicine, such as antiproton treatment of cancer.

The primary goal for antimatter research is not futuristic applications, but to uncover the symmetries (or possible asymmetries) of the fundamental laws of physics. One could expect that the matter and antimatter contents of the universe would be symmetric, but so far observations indicate that at least in the neighborhood of our galaxy there is only matter. Today the coexistence of matter and antimatter is ruled out on scales $\lesssim 20 \mathrm{Mpc}[1]$. The reason for this matter-antimatter asymmetry could be a violation of the CPT theorem. This

Email address: jonsell@tp.umu.se (S Jonsell). 
theorem, and other matter-antimatter symmetries, could be tested through high-precision measurements on antihydrogen. Two experiments at CERN, ATHENA and ATRAP, have succeeded in creating antihydrogen atoms, but much work remains before CPT tests can be carried out [3].

Despite the interests in antimatter of both scientists and the general public, relatively little is known about the interaction between atoms made of antimatter and ordinary atoms. So far no experimental data exist, and theoretical work has only just started. Atom-antiatom scattering has practical implications for antihydrogen experiments, e.g. unwanted scattering of antiatoms on impurities or on the walls of the experiment, or scattering on ultracold atoms introduced on purpose to achieve sympathetic cooling of antimatter. Atomantiatom scattering is also interesting in its own right, being a new fundamental process involving both atomic and low-energy nuclear physics.

A number of inelastic processes are possible in atom-antiatom scattering: nucleus-antinucleus annihilation, electron-positron annihilation, rearrangement processes (such as formation of positronium), and even formation of metastable "molecules". The most important of these are nucleus-antinucleus annihilation and rearrangement. Rearrangement processes will also eventually lead to annihilation, but on a time scale much longer than the time required for the final-state fragments to separate. Hence, it can be regarded as a process distinct from the "direct" annihilation during the collision. Alternatively, rearrangement can be regarded as a resonance in the annihilation channel. In this paper I will focus on the direct annihilation process, while rearrangement is discussed extensively elsewhere in these proceedings [4].

At large internuclear separations $R$ the wave function of the atom-antiatom system with relative angular momentum $l$ is given by

$$
\Phi\left(\boldsymbol{R}, \boldsymbol{r}_{i}\right) \rightarrow N \frac{1}{R} \sin \left(k_{i} R-l \pi / 2+\delta_{l}\right) Y_{l m}\left(\Omega_{R}\right) \psi_{\overline{\mathrm{H}}}\left(\boldsymbol{r}_{1}\right) \phi_{\mathrm{atom}}\left(\boldsymbol{r}_{i>1}\right),
$$

where $\psi_{\overline{\mathrm{H}}}$ and $\phi_{\text {atom }}$ are the atomic wave functions of antihydrogen and the atom, $\boldsymbol{r}_{i}$ denotes the coordinates of all leptons, and $\hbar k_{i}$ is the relative momentum in the initial state. Both elastic and annihilation cross sections are given by the complex phase shift $\delta_{l}[2]$,

$$
\begin{aligned}
\sigma^{\mathrm{el}} & =\frac{\pi}{k_{i}^{2}}(2 l+1)\left|1-e^{2 i \delta_{l}}\right|^{2}, \\
\sigma^{a} & =\frac{\pi}{k_{i}^{2}}(2 l+1)\left(1-e^{-4 \operatorname{Im} \delta_{l}}\right) .
\end{aligned}
$$

At low temperatures $(\lesssim 1 \mathrm{~K})$ only the $l=0$ partial wave contributes, and the phase shift has the low-energy form

$$
\lim _{k_{i} \rightarrow 0} \tan \delta_{0}=-k_{i} a,
$$


where $a=\alpha-i \beta$ is the complex scattering length. Inserting (4) into (2) and (3) gives the low-energy form of the cross sections

$$
\begin{aligned}
\sigma^{\mathrm{el}} & =4 \pi\left(\alpha^{2}+\beta^{2}\right), \\
\sigma^{a} & =\frac{4 \pi}{k_{i}} \beta .
\end{aligned}
$$

We first note that the elastic cross section is constant at low energies, while the annihilation cross section diverges as $k_{i}^{-1}$. Thus, below a certain energy annihilation dominates. Second, we note that $\sigma^{\text {el }}$ depends on both $\alpha$ and $\beta$. This means that a non-zero imaginary part $\beta$ of the scattering length, arising from the inclusion of the strong nuclear force, will not only give rise to annihilation, but will also significantly modify the elastic cross section. Only if the annihilation cross section is small, $\beta \ll|\alpha|$, this effect may be ignored.

The simplest way to calculate the annihilation cross section is to use a pseudo potential

$$
V_{a}(\boldsymbol{R})=A \delta(\boldsymbol{R})
$$

This is a contact interaction with strength given by the constant $A$. This constant can be determined from experimental data on the nucleus-antinucleus system, or it can be calculated from model potentials. Using the pseudo potential in first-order perturbation theory the annihilation cross section becomes

$$
\sigma^{a}=\frac{(2 \pi)^{3}}{k_{i}^{2}} A \lim _{R \rightarrow 0} \int\left|\Phi\left(\boldsymbol{R}, \boldsymbol{r}_{i}\right) / R\right|^{2} d \boldsymbol{r}_{i}
$$

For the proton-antiproton system $A^{p \bar{p}}=1.7 \times 10^{-7}$ a.u. $=6.8 \times 10^{-37} \mathrm{eVm}^{3}$, which was determined from the width $1130 \mathrm{eV}$ of the $1 s$ state of protonium [5]. For the alpha particle-antiproton system no data on the $1 s$ state are available, instead the value $A^{\alpha \bar{p}}=3.4 \times 10^{-7}$ a.u. $=1.4 \times 10^{-36} \mathrm{eVm}^{3}$ was determined from a combination of low-energy annihilation data, the energy shift of the $2 p$ state, and the width of the $2 p$ and $3 d$ state [6]. Both values represent an average over different spin states.

The pseudo-potential approach, being based on first-order perturbation theory, does not take into account the modification of the initial channel due to the strong force. Even if annihilation cannot be treated perturbatively one may still use the zero-range approximation of the strong force, since the atomic nucleus is very small on an atomic scale. The detailed shape of the strongforce potential will therefore not matter, only its strength as parameterized by a short-range strong-force phase shift $\delta_{s f}$. Furthermore, atomic energies (eV) are very small compared to typical nuclear energies $(\mathrm{MeV})$. The strong-force phase shift can therefore be parameterized by a strong-force scattering length $a_{s f}$. This scattering length is complex, with the imaginary part representing annihilation. 
The role of the strong-force scattering length can be understood by looking at the boundary condition of the wave function at short internuclear separations, $R \rightarrow 0$. In the absence of the strong force, the wave function has a shortdistance form proportional to the regular Coulomb function for zero angular momentum $F_{0}(k R)$. Even when the strong force is added the wave function is Coulombic for $R_{0} \leq R \ll 1 a_{0}$, where $R_{0}$ is just outside the range of the strong force. But now, since the Coulombic solution is not extended all the way to $R=0$, also the irregular Coulomb function $G_{0}(k R)$ is allowed. A general eigensolution to the zero-angular momentum Coulomb problem is an arbitrary linear combination of $F_{0}(k R)$ and $G_{0}(k R)$. For atom-antiatom scattering in the presence of the strong nuclear force the appropriate short-range form of the scattering function is given by a particular solution determined by the strong-force phase shift $\delta_{s f}$

$$
\Phi\left(\boldsymbol{R}, \boldsymbol{r}_{i}\right)=\frac{F_{0}(k R)+\tan \delta_{s f} G_{0}(k R)}{F_{0}\left(k R_{0}\right)+\tan \delta_{s f} G_{0}\left(k R_{0}\right)} \varphi\left(\boldsymbol{r}_{i}\right) .
$$

Here $\varphi\left(\boldsymbol{r}_{i}\right)$ is the wave function of the leptons in the combined electric field of the overlapping nucleus and antinucleus. The wave vector $k$ is related to $k_{i}$ as $\hbar^{2} k^{2} /(2 \mu)=\hbar^{2} k_{i}^{2} /(2 \mu)-E_{f}$, where $E_{f}$ is the leptonic energy corresponding to $\varphi\left(\boldsymbol{r}_{i}\right)$. Since $R_{0}$ is very small on an atomic scale, the boundary condition of $\Phi$ at $R_{0}$ may be expressed in terms of the short-range expansions of the Coulomb functions [8],

$$
\begin{aligned}
F_{0}(k R) & \rightarrow C_{0}(\eta) k R, \\
G_{0}(k R) & \rightarrow C_{0}^{-1}(\eta)\{1-2 \eta k R[\ln (2 \eta k R)+h(\eta)+2 \gamma-1]\} .
\end{aligned}
$$

Here the strength of the Coulomb interaction is given by $\eta=1 /\left(b_{\mu} k\right)$, where $b_{\mu}=a_{0} m_{e} / \mu$ is the mass-scaled Bohr radius. The normalization constant is $C_{0}(\eta)=\sqrt{2 \pi \eta /[1-\exp (-2 \pi \eta)]}, \gamma \simeq 0.5772 \ldots$ is the Euler constant, and $h$ can be expressed in terms of the digamma function $\Psi$ as

$$
h(\eta)=[\Psi(-i \eta)+\Psi(i \eta)] / 2-\ln \eta .
$$

One then finds that [9]

$$
\frac{C_{0}^{2}(\eta)}{\eta} \cot \delta_{s f}-2 h(\eta)=-\frac{b_{\mu}}{a_{s f}}
$$

where the strong-force scattering length is

$$
\frac{1}{a_{s f}}=-\frac{2 \pi}{b_{\mu}} \lim _{k \rightarrow 0} \cot \delta_{s f}
$$

The wave function $\Phi$ may be expressed in terms of a regular and an irregular 
solution as

$$
\begin{aligned}
\Phi\left(\boldsymbol{R}, \boldsymbol{r}_{i}\right)= & -\frac{b_{\mu}^{2} /\left(a_{s f} R_{0}\right) F_{0}\left(k R_{0}\right)}{\left[2 h(\eta)-b_{\mu} / a_{s f}\right] F_{0}\left(k R_{0}\right)+C_{0}(\eta)^{2} / \eta G_{0}\left(k R_{0}\right)} \Phi^{\mathrm{reg}}\left(\boldsymbol{R}, \boldsymbol{r}_{i}\right) \\
& +\frac{2 h(\eta) F_{0}\left(k R_{0}\right)+C_{0}^{2}(\eta) / \eta G_{0}\left(k R_{0}\right)}{\left[2 h(\eta)-b_{\mu} / a_{s f}\right] F_{0}\left(k R_{0}\right)+C_{0}(\eta)^{2} / \eta G_{0}\left(k R_{0}\right)} \Phi^{\text {irreg }}\left(\boldsymbol{R}, \boldsymbol{r}_{i}\right) .
\end{aligned}
$$

The regular and irregular solutions used are defined by their short-range forms

$$
\begin{aligned}
\lim _{R \rightarrow 0} \Phi^{\mathrm{reg}}\left(\boldsymbol{R}, \boldsymbol{r}_{i}\right) & =\frac{R_{0}}{b_{\mu} F_{0}\left(k R_{0}\right)} F_{0}(k R) \varphi\left(\boldsymbol{r}_{i}\right) \simeq \frac{R}{b_{\mu}} \varphi\left(\boldsymbol{r}_{i}\right) \\
\lim _{R \rightarrow 0} \Phi^{\operatorname{irreg}}\left(\boldsymbol{R}, \boldsymbol{r}_{i}\right) & =\frac{1}{\frac{2 \eta h(\eta)}{C_{0}(\eta)^{2}} F_{0}\left(k R_{0}\right)+G_{0}\left(k R_{0}\right)}\left[\frac{2 \eta h(\eta)}{C_{0}(\eta)^{2}} F_{0}(k R)+G_{0}(k R)\right] \varphi\left(\boldsymbol{r}_{i}\right) \\
& \simeq\left\{1-\frac{2 R}{b_{\mu}}\left[\ln \left(\frac{2 R}{b_{\mu}}\right)+2 \gamma-1\right]\right\} \varphi\left(\boldsymbol{r}_{i}\right) .
\end{aligned}
$$

These forms have the advantage that they are independent of $R_{0}$, provided that $R_{0}$ is small enough. The normalization factors ensure that $\Phi^{\text {reg }}$ and $\Phi^{\text {irreg }}$ are real functions, even if $k$ is not. For large internuclear separations

$$
\begin{aligned}
\lim _{R \rightarrow \infty} \Phi^{\text {reg }}\left(\boldsymbol{R}, \boldsymbol{r}_{i}\right) & =N_{\text {reg }} \frac{1}{R} \sin \left(k_{i} R+\delta_{\text {reg }}\right) \psi_{\overline{\mathrm{H}}}\left(\boldsymbol{r}_{1}\right) \phi_{\text {atom }}\left(\boldsymbol{r}_{i>1}\right), \\
\lim _{R \rightarrow \infty} \Phi^{\text {irreg }}\left(\boldsymbol{R}, \boldsymbol{r}_{i}\right) & =N_{\text {irreg }} \frac{1}{R} \sin \left(k_{i} R+\delta_{\text {irreg }}\right) \psi_{\overline{\mathrm{H}}}\left(\boldsymbol{r}_{1}\right) \phi_{\text {atom }}\left(\boldsymbol{r}_{i>1}\right),
\end{aligned}
$$

where both normalization factors and phase shifts are real. Inserting (18) and (19) into (15), and using (1), the complex phase shift $\delta_{0}$ for atom-antiatom scattering including the strong nuclear force is found to be

$$
\cot \delta_{0}=\frac{N_{\text {reg }} \cos \delta_{\text {reg }}-a_{s f} / b_{\mu} N_{\text {irreg }} \cos \delta_{\text {irreg }}}{N_{\text {reg }} \sin \delta_{\text {reg }}-a_{s f} / b_{\mu} N_{\text {irreg }} \sin \delta_{\text {irreg }}} .
$$

Here the approximation $k R_{0} \rightarrow 0$ has been used. With this equation we arrive at a very simple way of calculating the strong-force effects beyond the pseudo potential in first-order perturbation theory. In addition to the usual regular solution (16), an additional irregular solution given by the boundary condition (17) has to be calculated. The asymptotic forms of the two solutions is then weighted according to (20) using the strong-force scattering length.

The scattering length method has several advantages. It is very easy to implement numerically. It makes no assumptions about the detailed form of the strong-force potential, and is hence model independent. Alternatively one may directly use Eq. (9), in combination with Eq. (13), as a short-range boundary condition, and integrate towards $R=\infty$. This was the procedure in Refs. $[7,13,16]$. The present formulation in terms of Eq. (20) has the advantage that it clearly brings out the relation between the atom-antiatom phase shift $\delta_{0}$ 
and the strong-force scattering length $a_{s f}$, in terms of a minimal number of parameters. The parameters $N_{\text {reg }}, N_{\text {irreg }}, \delta_{\text {reg }}$, and $\delta_{\text {irreg }}$ are independent of the strong force. Once they have been determined from an atom-antiatom calculation, it is a trivial task to calculate $\delta_{0}$ for a given strong-force scattering length.

Using this method elastic and annihilation cross sections have been calculated for $\mathrm{H}-\overline{\mathrm{H}}$ and $\mathrm{He}-\overline{\mathrm{H}}$. The strong-force scattering lengths used were $a_{s f}^{p \bar{p}}=$ $(0.844-0.698 i) \mathrm{fm}$, and $a_{s f}^{\alpha \bar{p}}=(1.851-0.630 i) \mathrm{fm}$, determined from the same data as the annihilation constants $A^{p \bar{p}}$ and $A^{\alpha \bar{p}}$ above. Once again, these are averages over different spin states. The $\mathrm{H}-\overline{\mathrm{H}}$ potential was taken from Ref. [10] and the $\mathrm{He}-\overline{\mathrm{H}}$ potential from Ref. [11]. In figures 1 and 2 we compare the pseudo-potential and the scattering-length approaches for $\mathrm{H}-\overline{\mathrm{H}}$ and $\mathrm{He}-\overline{\mathrm{H}}$ respectively.

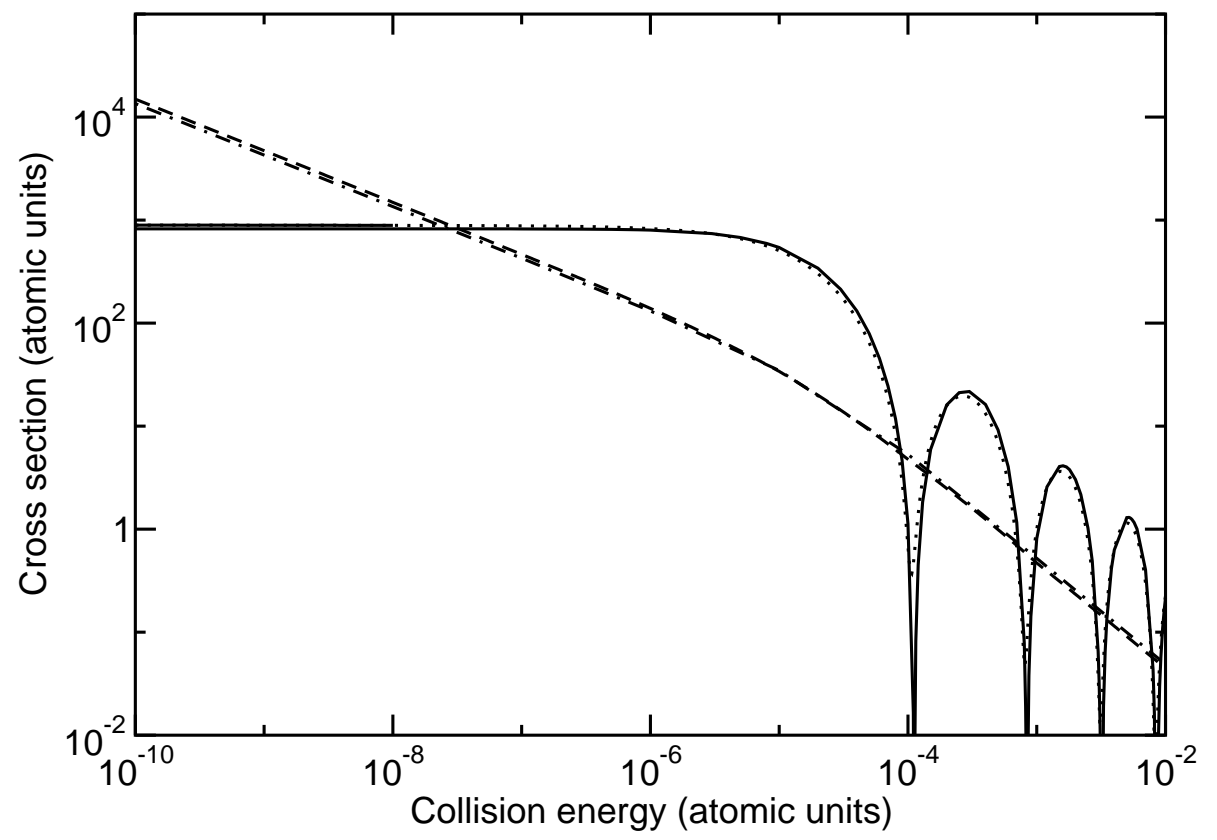

Fig. 1. Cross sections for $\mathrm{H}-\overline{\mathrm{H}}$ scattering; (solid) elastic cross section including the strong force, (dotted) elastic cross section without the strong force, (dashed) annihilation using the scattering length approach (dash-dotted) annihilation using pseudo potential. Only the $s$-wave part of the elastic cross section is shown.

For $\mathrm{H}-\overline{\mathrm{H}}$ the pseudo-potential approach works quite well. As has been reported earlier, the low-energy annihilation cross section using the pseudopotential approach is $0.14 / \sqrt{\epsilon_{i}} a_{0}^{2}$ [12], while the scattering length approach gives $0.15 / \sqrt{\epsilon_{i}} a_{0}^{2}$ [13] $\left(\epsilon_{i}\right.$ is the collision energy). This is also in good agreement with a calculation by Armour et al. [14],using the optical potential of Kohno and Weise [15], who obtained $0.12 / \sqrt{\epsilon_{i}} a_{0}^{2}$ for the triplet state and $0.15 / \sqrt{\epsilon_{i}} a_{0}^{2}$ for the singlet state. The elastic cross section changes from $771 a_{0}^{2}$ to $892 a_{0}^{2}$ when the strong-force effects are included. The change obtained by Armour et al. [14] was from $788 a_{0}^{2}$ to $920 a_{0}^{2}$ for the triplet and $872 a_{0}^{2}$ for the 
singlet. The scattering length is $a=(8.4-0.51 i) a_{0}$, so indeed $\beta \ll|\alpha|$ as required for the validity of the pseudo-potential approach.

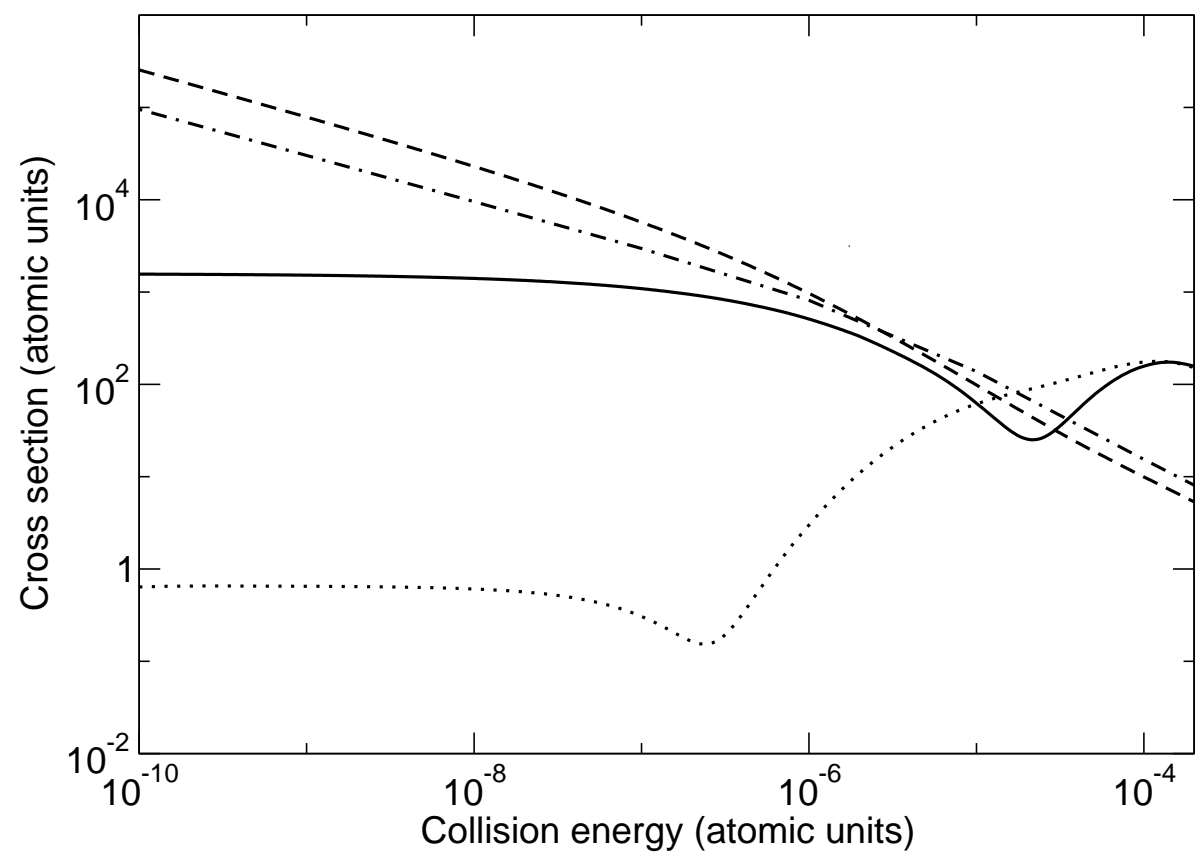

Fig. 2. Cross sections for $\mathrm{He}-\overline{\mathrm{H}}$ scattering; (solid) elastic cross section including the strong force, (dotted) elastic cross section without the strong force, (dashed) annihilation using the scattering length approach (dash-dotted) annihilation using pseudo potential.

For $\mathrm{He}-\overline{\mathrm{H}}$ scattering the situation is quite different. The strong force has a drastic influence on the elastic cross section. This is in part because the real part of the scattering length happens to have an untypically low value $\alpha=-0.23 a_{0}$ (the typical scale of the scattering length is $\sim 5 a_{0}$ ). The elastic cross section without the strong force is therefore only $0.65 a_{0}$, but changes drastically to $1700 a_{0}^{2}$ when the strong force is included. This is reflected by the scattering length $a=(2.2-11.4 i) a_{0}$, which has an imaginary part much larger than the real part. The annihilation cross section changes from $0.95 / \sqrt{\epsilon_{i}} a_{0}^{2}$ in the pseudo-potential approach to $2.62 / \sqrt{\epsilon_{i}} a_{0}^{2}$ in the scattering-length approach.

These results replace those in Ref. [16] which were based on the potential in Ref. [17]. This potential had been calculated at fewer $R$-values, giving interpolation problems in the cross section calculations. Rather surprisingly, the effect of this refinement of the potential on both elastic and annihilation cross sections was quite large. The scattering length reported in Ref. [16] was $a=(-7.69-3.80 i) a_{0}$, giving an annihilation cross section $0.88 / \sqrt{\epsilon_{i}} a_{0}^{2}$. The increase of the calculated annihilation cross section with a factor three can almost entirely be attributed to the range $R \lesssim 1.5 a_{0}$ of the potential. The difference between the old and the new potential is very small, at most about 0.007 a.u. (at $R=0.8 a_{0}$ ), showing the extreme sensitivity of the scattering 
length to the details of the potential. Although this is a small correction, it is still 20 times larger than the maximum of the adiabatic correction [11], which on the contrary only has a small impact on the scattering length.

The validity of the scattering length approach has been tested for the $\mathrm{H}-\overline{\mathrm{H}}$ system using a model potential with the form [6]

$$
V_{\text {opt }}(R)=-\frac{2 \pi \hbar^{2}}{\mu}\left(1+\frac{A-1}{A} \frac{\mu}{m}\right) b_{0} \rho(R) .
$$

Here $A$ is the nuclear mass number, $b_{0}$ a complex parameter, $\mu$ the antiprotonnucleon reduced mass, $m$ the mass of the nucleon, and $\rho(R)$ the nuclear mass density, which was taken to have a Gaussian form with width $r_{G}$. Three different widths were used, $r_{G}=1.0,1.5,2.0 \mathrm{fm}$, and for each width the parameter $b_{0}$ was adjusted until the potential reproduced the scattering length above. All three potentials thus obtained gave results identical to the scattering-length approach, to within three significant digits [7]. This clearly shows that the shape of the potential does not matter, and all its relevant properties can be summarized by the complex strong-force scattering length $a_{s f}$.

In conclusion I have developed a simple method to take the strong force into account in situations when the pseudo-potential method cannot be applied. This is the case in He- $\overline{\mathrm{H}}$ scattering, where annihilation is fast, and the elastic cross section is drastically modified by the strong force. In the future this method may be used to investigate scaling properties of elastic and annihilation cross sections for scattering of antihydrogen on heavier atoms.

\section{References}

[1] A. G. Cohen, A. De Rújula, S. L. Glashow, Astrophys. J. 495 (1998) 539.

[2] L. D. Landau, E. M. Lifshitz, Quantum Mechanics, 3rd edn. (ButterworthHeinemann, Oxford, 1977)

[3] M. Amoretti et al., Nature 419 (2002) 456; G. Gabrielse et al., Phys. Rev. Lett. 89 (2002) 213401; G. Gabrielse et al., Phys. Rev. Lett. 89 (2002) 233401.

[4] E. A. G. Armour, S. Jonsell, Y. Liu, A. C. Todd, these proceedings.

[5] C. J. Batty, Nucl. Phys. A 601 (1996) 425.

[6] C. J. Batty, E. Friedman, A. Gal, Nucl. Phys. A 689 (2001) 721.

[7] S. Jonsell, A. Saenz, P. Froelich, B. Zygelman, A. Dalgarno, Canadian J. Phys. 83 (2005) 435.

[8] M. Abramowitz, and I. A. Stegun, Handbook of Mathematical Functions, New York, Dover (1964). 
[9] T. L. Trueman, Nucl. Phys. 26 (1961) 57.

[10] K. Strasburger, J. Phys. B: At. Mol. Opt. Phys. 35 (2002) L435.

[11] K. Strasburger, H. Chojnacki, A. Sokolowska, J. Phys. B: At. Mol. Opt. Phys. 38 (2005) 3091.

[12] S. Jonsell, A. Saenz, P. Froelich, B. Zygelman, A. Dalgarno, Phys. Rev. A 64 (2001) 052712.

[13] S. Jonsell, A. Saenz, P. Froelich, B. Zygelman, A. Dalgarno, J. Phys. B: At. Mol. Opt. Phys. 37 (2004) 1195.

[14] E. A. G. Armour et al. J. Phys. B: At. Mol. Opt. Phys. 38 (2005) L47.

[15] M. Kohno, W. Weise, Nucl. Phys. A 454 (1986) 429.

[16] S. Jonsell, P. Froelich, S. Eriksson, K. Strasburger, Phys. Rev. A 70 (2004) 062708 .

[17] K. Strasburger, H. Chojnacki, Phys. Rev. Lett. 88 (2002) 163201. 